\title{
Perceptions des acteurs sur le financement des services agricoles au Bénin
}

Stakeholders' perceptions on the financing of agricultural research and extension in Benin

Ismail M. Moumouni

\section{(2) OpenEdition}

Journals

Édition électronique

URL : http://journals.openedition.org/economierurale/3915

DOI : 10.4000/economierurale.3915

ISSN : 2105-2581

Éditeur

Société Française d'Économie Rurale (SFER)

Édition imprimée

Date de publication : 15 mars 2013

Pagination : 69-83

ISSN : 0013-0559

Référence électronique

Ismail M. Moumouni, «Perceptions des acteurs sur le financement des services agricoles au Bénin », Économie rurale [En ligne], 334 | mars-avril 2013, mis en ligne le 15 mars 2015, consulté le 28 janvier 2020. URL : http://journals.openedition.org/economierurale/3915; DOI : 10.4000/economierurale. 3915

(c) Tous droits réservés 


\title{
Perceptions des acteurs sur le financement des services agricoles au Bénin
}

\author{
Ismail M. MOUMOUNI • Faculté d'Agronomie, Université de Parakou, Bénin \\ ismailmm@gmail.com
}

Cet article analyse les perceptions des acteurs sur le financement de la recherche et de la vulgarisation agricoles au Bénin. La plupart des acteurs pensent que ce financement devrait provenir principalement de l'État. Les producteurs accepteraient d'y apporter une contribution si les filières agricoles étaient organisées. II existe cependant des facteurs socio-économiques qui influencent négativement la reconnaissance de la valeur marchande des services.

MOTS-CLÉS : Bénin, financement, recherche, vulgarisation, perception des acteurs

\section{Stakeholders' perceptions on the financing of agricultural research and extension in Benin}

This paper analyzes stakeholders' on the financing of these agricultural research and extension services in Benin. Stakeholders assume that the Government must mainly finance these services. Farmers would agree to contribute a share if commodity networks are well-organized. However, some socio-economic factors negatively influence the recognition of the commercial value of services. (JEL: Q16, Q18, 017)

KEYWORDS: Benin, Extension, Financing, Research, Stakeholders' Perceptions

D ans les projets de développement agricole, la participation est souvent définie comme l'implication des producteurs ou des communautés rurales dans les processus de planification du développement agricole (Nouatin, 2003). Un tel partenariat devrait être caractérisé par une confiance mutuelle et constituer un environnement favorable pour la conception et la diffusion de résultats efficaces, durables et à large impact (Schmidt et al., 1998). La participation est donc perçue comme un moyen d'augmenter les chances de succès des projets de développement agricole. Jusqu'au début des années 1990, la participation des communautés locales dans la Recherche et vulgarisation agricoles (RVA) avait été suscitée beaucoup plus dans l'organisation des services jadis offerts presque gratuitement aux producteurs dans les Pays en développement. À la suite de la libéralisation des services agricoles qui a conduit à un retrait partiel de l'État de l'organisation et du financement de la RVA, on constate que la Participation financière des producteurs (PFP) est de plus en plus sollicitée, encouragée et même parfois exigée. Le mécanisme par lequel les services de RVA sont financés affecterait leurs objectifs, groupes cibles, méthodes, messages et organisation. Les acteurs financent des services parce qu'ils estiment que c'est un moyen d'atteindre leurs buts (Ban, 2000).

Selon Katz (2002), la PFP peut développer un sens de responsabilité et d'intérêt au niveau des producteurs. Ainsi, si les producteurs engagent des dépenses pour soutenir la RVA, ils chercheraient à orienter les services vers la satisfaction de leurs besoins. Cette supposition a généré au cours de ces dernières années des stratégies de réformes diverses de la RVA en Afrique au sud du Sahara. On peut citer entre 
autres la commercialisation, la contractualisation, le recouvrement des coûts de la RVA (Katz, 2002 ; Rivera et Zijp, 2000 ; Rivera et Cary, 1997). La théorie économique des biens et services de Umali et Schwartz (1994) a constitué un cadre théorique légitimant les processus de privatisation. Elle se base sur les possibilités d'exclusion (excludability) et de rivalité (subtractability) que présentent les biens et services pour les classer en quatre types à frontière intangible. Les biens ayant un fort caractère privé pourraient faire l'objet d'une exploitation commerciale. Pendant que l'État et les organisations à but non lucratif s'occupent de biens et de services publics, les entreprises privées devraient fournir les biens et les services privés aux producteurs. Les services de RVA sont généralement considérés, selon cette théorie, comme des biens à péage à court terme et des biens publics à long terme (Umali et Schwartz, 1994 ; Carney, 1998). Cependant, les services de RVA ont été des objets de privatisation aussi bien dans les pays du Nord que du Sud. Les processus de privatisation ont été dans leur ensemble critiqués pour leurs effets destructeurs sur la cohésion sociale au sein des communautés locales, sur la gestion collective des systèmes de connaissances agricoles (Moumouni, 2007 ; Klerkx et Leeuwis, 2009). Ces observations posent, avec une grande importance, la question du financement de la RVA dont la consistance et la durabilité demeurent dans la plupart des Pays en développement des préoccupations brûlantes (Groupe de Neuchâtel, 2002). Dans ces conditions, il paraît nécessaire de s'intéresser aux perceptions des acteurs locaux et nationaux afin de les mettre en perspective avec les discours internationaux des institutions et bailleurs de fonds qui voient en la PFP un moyen d'assurer l'efficacité et la durabilité des services de RVA. L'évolution de la RVA au Bénin, similaire à celle des autres pays d'Afrique au sud du Sahara, constitue un cas intéressant pour une telle analyse. Jusqu'en 1991, les services de RVA, basés sur le système formation et visites, étaient fournis presque gratuitement aux producteurs par des institutions publiques, notamment l'Institut national des recherches agricoles et les Centres d'action régionale pour le développement rural (CARDER) (MDR, 1991, 1999). Les lettres de déclaration de la politique de développement rural de 1991 et de 1999 ont consacré et renforcé le processus de libéralisation. Depuis lors, des réformes sont en cours en vue d'améliorer l'efficacité et l'efficience de la RVA au Bénin (MAEP, 2001 ; MAEP, 2004 ; INRAB/MAEP, 2004). Il y eut un changement dans le mode de financement de la RVA. La participation de l'État se limite essentiellement aux frais de personnel et de fonctionnement des organismes publics impliqués. Les partenaires au développement financent la RVA à travers des projets de recherche ou de développement agricole. Les autres acteurs, y compris les producteurs, sont invités à apporter leur contribution.

Cet article a pour objectif, non pas de quantifier les contributions des acteurs, mais d'analyser leurs perceptions du financement de services de RVA au Bénin et de les mettre en perspective avec les discours internationaux sur la PFP. De façon plus spécifique, il vise à (i) identifier les sources actuelles de financement, (ii) analyser ce que les acteurs locaux et nationaux estiment devoir être les sources de financement de la RVA, (iii) étudier les obstacles et les conditions d'amélioration de la PFP suivant les perspectives des acteurs locaux et enfin, (iv) examiner, à la lumière des résultats, les relations entre la PFP et l'appropriation des services par les producteurs d'une part, et le caractère privé d'un service d'autre part. 


\section{Cadre théorique et méthodologique}

\section{Approche théorique et conceptuelle}

Cette recherche a été conduite principalement en utilisant une approche orientée vers les acteurs. Cette approche permet d'analyser les actions et réactions des individus/groupes dans les systèmes d'innovations institutionnelles et technologiques dans lesquels ils sont impliqués (Long, 1992). Cette excellente approche permet la déconstruction des interventions de développement (Long, 2003) et la PFP dans ce cas. Les populations supposées bénéficiaires des services de développement n'ont pas les mêmes perceptions des réalités que les agences d'intervention. L'hétérogénéité et le polymorphisme de la vie sociale justifient les différences de perceptions qu'il convient alors de prendre en considération dans la conception et la mise en œuvre des réformes et des projets de développement. L'approche orientée vers les acteurs permet de mieux comprendre comment les conditions socioprofessionnelles des acteurs influencent leurs perceptions et interprétations de la PFP. Nous désignons par perception le processus de pensée sur soi-même, sur les autres et les situations, c'est-à-dire comment l'on comprend et donne un sens à des stimuli environnementaux. Chaque acteur en fonction de son cadre de référence (Ban et al., 1994) se fait une compréhension de l'organisation et du financement de la RVA. La recherche agricole est définie ici comme toute forme d'investigation ou d'activité scientifique visant à identifier, comprendre ou résoudre un ou des problème(s) d'ordre agricole. Elle s'étend donc de la recherche thématique ou fondamentale à la recherche appliquée ou d'adaptation. La recherche agricole prend aussi en compte la recherche socioéconomique et/ou diagnostic dans ces secteurs. La vulgarisation inclut l'information, le transfert de technologie, la formation, l'assistance à la résolution de problèmes et la facilitation dans le secteur agricole (Groupe de Neuchâtel, 1999 ; Katz, 2002). La recherche et la vulgarisation apparaissent comme des fonctions sœurs du fait des interrelations particulières qui les lient. Elles sont non seulement complémentaires mais aussi se chevauchent. Il semble donc indiqué d'aborder la question de leur financement, pas de façon séparée, mais globalement. Le financement de la RVA est toute forme de contribution en nature ou en espèce en vue d'encourager ou de supporter une institution de services ou ses agents. Le concept de services agricoles se réfère dans ce contexte à la RVA.

\section{Approche méthodologique}

Une cinquantaine d'entretiens semi-structurés a été conduite : 45 (voir tableau 1) avec des personnes impliquées dans l'organisation des services de la RVA à divers niveaux (personnel dirigeant, agents de terrain) et cinq producteurs individuels.

Trois principales questions ont été abordées au cours des entretiens : (1) Quelle est votre politique de financement de la recherche et/ou de la vulgarisation agricoles ? (2) Quelles devraient être selon vous les sources de financement de la recherche et de la vulgarisation au Bénin ? (3) Que pensez-vous de la PFP dans l'organisation des services de RVA au Bénin ? Nous avons abordé cette dernière question en termes de perceptions des acteurs sur (i) les obstacles au financement par les paysans, (ii) les conditions d'augmentation de la participation financière paysanne et (iii) les formes de contribution des paysans. Les interviews ont été enregistrées, transcrites et analysées à l'aide du logiciel Atlas.ti qui a permis de faire ressortir les grandes tendances de perceptions. Les citations les plus expressives et les idées récurrentes des interviewés ont été rapportées comme " évidences, illustrations et pour faire entendre leur voix. » Nous avons catégorisé ces citations dans 
Benin : financement de la recherche et vulgarisation agricoles

Tableau 1. Nombre de personnes interviewées par type d'organisation

\begin{tabular}{l|c|c|c}
\hline \multicolumn{1}{c|}{ Types d'organisation } & $\begin{array}{c}\text { Membres } \\
\text { du personnel dirigeant }\end{array}$ & $\begin{array}{c}\text { Agents de } \\
\text { terrain }\end{array}$ & Total \\
\hline $\begin{array}{l}\text { Organisations ministère de l'Agriculture publiques } \\
\text { université, institutions publiques de recherche et } \\
\text { de vulgarisation }\end{array}$ & 9 & 8 & 17 \\
\hline Institutions semi-publiques (Projets et programmes) & 5 & 4 & 9 \\
\hline Organisations non gouvernementales & 5 & 4 & 9 \\
\hline Organisations paysannes & 4 & 3 & 7 \\
\hline Organisations interprofessionnelles & 3 & - & 3 \\
\hline Total & 26 & 19 & 45 \\
\hline
\end{tabular}

Source : I'auteur.

des tableaux à double entrée suivant la nature des institutions de provenance des interviewés (ministère, université, recherche, vulgarisation, ONG, organisation de producteurs, projet de développement) et de leur niveau de hiérarchie professionnelle (membres du personnel dirigeant ou agents de terrain). Cela a permis de détacher les évidences provenant des différentes perspectives ou catégories d'acteurs.

\section{Sources actuelles de financement}

\section{Financement par les bailleurs étrangers}

Les bailleurs de fonds étrangers sont une source importante de financement de la RVA au Bénin. La recherche publique réunit des fonds (1) des institutions internationales de recherche telles que l'Institut international d'agriculture tropicale, le Centre de coopération internationale en recherche agronomique pour le développement, l'Association pour le développement de la riziculture en Afrique de l'Ouest, (2) de coopérations bilatérales avec des pays comme le Danemark, les Pays-Bas, l'Allemagne et la France. Ces fonds sont souvent investis sous forme de projets de recherche ou de développement tels que le Programme spécial pour la sécurité alimentaire et le Programme de développement des plantes à racines et tubercules. Des projets de développement agricoles sont aussi financés par des institutions internationales telles que la Banque mondiale, le Fonds international pour le développement agricole, l'Organisation des Nations unies pour l'alimentation et l'agriculture, la Banque ouestafricaine de développement. Les ONG bénéficient du soutien financier des projets pour fournir des services de proximité aux producteurs.

\section{Financement public}

L'État contribue insuffisamment au fonctionnement des projets et programmes de RVA. Cependant, il a toujours conservé son infrastructure organisationnelle de vulgarisation. Mais ces dernières années, le nombre d'agents en activité est devenu très insuffisant pour répondre aux sollicitations des producteurs. Les mairies sont supposées participer à l'organisation et au financement des services de RVA sur leur territoire. Mais elles ont encore des difficultés à supporter pleinement leurs charges de fonctionnement. 
RECHERCHES

Ismail M. MOUMOUNI

\section{Financements privés.}

\section{Paysans, organisations et autres acteurs}

Les producteurs sont souvent sollicités pour apporter une contribution au financement de la recherche ou de la vulgarisation dans le cadre des projets de développement. Cette contribution est le plus souvent matérielle mais aussi parfois financière.

\section{Financement en espèce}

Le financement en espèce des producteurs au profit de la RVA est encore très limité. Ces dernières années, le financement de la RVA a été marqué par l'implication de l'Association interprofessionnelle du coton (AIC) au Bénin. Cette association constituée par l'Association nationale des producteurs de coton, le Groupement des professionnels de distribution des intrants agricoles et l'Association des professionnels d'égrenage du Bénin - apporte une contribution substantielle au financement de fonctions dites critiques que sont la recherche, la vulgarisation et la réalisation des pistes rurales. Ces fonds sont constitués par des prélèvements de $10 \mathrm{FCFA} /$ tonne de coton graine vendue par le producteur et sont utilisés pour renforcer les services publics en ressources humaines et matérielles. De nombreuses ONG contribuent aussi à mobiliser le financement pour la formation des producteurs. Ainsi, des expériences encore isolées de financement en espèce existent. Il s'agit le plus souvent du financement des formations ponctuelles ou de longue durée.

\section{Financement en nature}

Quatre principaux types de financement en nature sont observés :

- lopins de terre et main-d'œuvre : des producteurs offrent des lopins de terre, leur force de travail et parfois des intrants pour la conduite des expérimentations ;

- produits de récoltes : en vue d'encourager les agents d'encadrement, les producteurs sont souvent prêts à leur offrir des produits de récoltes ;

- matériaux locaux : gravier, sable, bois et autres matériaux, par exemple pour la construction de poulaillers d'expérimentation, de magasins de stockage, etc.;

- temps d'encadrement : des producteurs leaders sont choisis dans chaque village et formés pour former d'autres producteurs sans ou avec une symbolique contrepartie financière.

Le financement en nature n'est pas systématique et le plus souvent il n'est pas pris en compte dans les budgets prévisionnels ou les évaluations. De plus, il constitue souvent une motivation supplémentaire pour les agents et non un financement de l'institution à laquelle ils appartiennent. L'intégration des producteurs leaders dans les dispositifs d'encadrement permet d'augmenter la couverture de celui-ci et de réduire son coût. L'intégration est aussi perçue comme une étape essentielle dans la pérennisation des acquis des projets. Selon la plupart des acteurs, la contribution en nature n'est pas toujours un signe d'un intérêt réel des producteurs dans la RVA, cela peut être une source de prestige social et permet de bénéficier des avantages des projets de développement.

Après cette revue de contexte, nous examinons dans la section suivante ce que devraient être les sources de financement de la RVA selon les acteurs.

\section{Sources de financement de la recherche et de la vulgarisation selon les acteurs}

\section{1. État}

Tous les acteurs consultés pensent que l'État devrait assurer le principal du financement de la recherche et de la vulgarisation. Deux raisons sont avancées : 
- La nécessité de garantir la souveraineté de l'État dans la mise en œuvre de la politique agricole : la recherche ne devrait pas être laissée aux seuls financements des entreprises privées et des institutions internationales dont les intérêts ne sont pas toujours en harmonie avec ceux de l'État et des populations. L'État devrait donc apporter sa contribution pour ne pas laisser un secteur aussi important échapper à son contrôle.

- La rentabilité à long terme de la RVA : la RVA n'est pas constituée d'activités rentables à court terme pour les acteurs privés. On ne saurait demander à un acteur privé de financer un travail dont tout le monde utiliserait le résultat. Mais toute activité qui est de l'intérêt de la communauté nationale est de celui de l'État. Il revient donc à l'État d'intervenir dans de tels secteurs où les acteurs individuels ne trouvent pas leur compte ou n'en perçoivent pas la portée.

En somme, le budget national devrait être le socle du financement de la RVA pour tous les acteurs. Ce financement public reste indispensable. Ceci est d'autant plus important que la recherche fondamentale est presque occultée actuellement au profit de la recherche appliquée. Selon les acteurs, notamment ceux des services publics de recherche et de vulgarisation, il serait donc important de constituer un fonds pour rendre durable et autonome le mécanisme de fonds compétitifs de recherche.

\section{Projets ou bailleurs de fonds étrangers}

Pour le personnel dirigeant des institutions de RVA, le financement des bailleurs de fonds étrangers devrait venir seulement en complément de celui de l'État et pour soutenir les efforts de mobilisation de ressources des acteurs locaux et nationaux. La plupart des dirigeants des ONG estiment que le financement par des partenaires étrangers sera toujours indispensable. Par contre, pour les agents des projets de développement, ce financement étranger devrait être considéré comme complémentaire à celui de l'État. Mais beaucoup partagent la crainte que les efforts nationaux de mobilisation de financement de la RVA soient inhibés par les financements extérieurs. Même s'ils admettent que le financement extérieur est aujourd'hui nécessaire, les acteurs locaux s'accordent à dire qu'il faut développer des sources alternatives plus durables.

\section{Opérateurs privés}

Comme à travers l'Association interprofessionnelle du coton, des opérateurs privés devraient s'impliquer dans l'organisation et le financement de la RVA en vue de promouvoir d'autres filières porteuses. Aussi, ils pourraient commanditer des travaux de recherche dont ils utiliseraient les résultats pour améliorer leurs performances. Il est cependant nécessaire de remarquer que les intérêts des opérateurs privés et ceux de l'État ne coïncident pas toujours. Par exemple, les distributeurs d'intrants ne seraient pas motivés à financer des technologies utilisant moins d'engrais ou de pesticides. D'un autre point de vue, ces distributeurs auraient intérêt à financer des activités permettant aux producteurs de découvrir et de bien apprécier leurs produits afin de mieux les vendre.

\section{Les producteurs et leurs organisations}

Les perceptions des acteurs sur le financement par les producteurs et leurs organisations de la RVA sont en grande partie convergentes (tableau 2).

Les reformes de privatisation en cours visent à faire participer les producteurs au financement de la recherche et de la vulgarisation. Les membres du personnel dirigeant des organisations spécifient les aspects qui devraient bénéficier du 
RECHERCHES

Ismail M. MOUMOUNI

Tableau 2. Synthèse des perceptions des différents types d'acteurs sur le financement de la RVA par les producteurs et leurs organisations

\begin{tabular}{|c|c|c|}
\hline & Membres du personnel dirigeant & Agents de terrain \\
\hline Ministère & $\begin{array}{l}\text { Avec la nouvelle politique de développement agricole, } \\
\text { nous tendons vers une plus grande participation } \\
\text { financière des producteurs dans la recherche-dévelop- } \\
\text { pement surtout. }\end{array}$ & - \\
\hline Université & $\begin{array}{l}\text { Certains gros producteurs sont plus ou moins des } \\
\text { entreprises agricoles et peuvent, dans le cadre de leurs } \\
\text { associations, financer des services de RVA. }\end{array}$ & - \\
\hline $\begin{array}{l}\text { Recherche } \\
\text { publique }\end{array}$ & $\begin{array}{l}\text { Les organisations paysannes faîtières devraient travail- } \\
\text { ler avec la recherche et la financer pour trouver des } \\
\text { solutions à des problèmes concrets. }\end{array}$ & $\begin{array}{l}\text { Les organisations paysannes } \\
\text { faîtières devraient financer la } \\
\text { recherche et la vulgarisation. }\end{array}$ \\
\hline $\begin{array}{l}\text { Vulgarisation } \\
\text { publique }\end{array}$ & $\begin{array}{l}\text { Au regard des réformes en cours où un accent est mis } \\
\text { sur le développement des filières, les OP sont appelées } \\
\text { à investir davantage dans la RVA. }\end{array}$ & $\begin{array}{l}\text { Si les producteurs veulent voir } \\
\text { leurs performances et revenus } \\
\text { agricoles améliorés, ils devraient } \\
\text { pouvoir financer les services } \\
\text { agricoles. }\end{array}$ \\
\hline ONG & $\begin{array}{l}\text { Quand les produits de recherche arrivent à maturité, } \\
\text { les producteurs devraient pouvoir assurer la conti- } \\
\text { nuité, c'est-à-dire la diffusion. }\end{array}$ & $\begin{array}{l}\text { " Il faudrait soulever sa charge } \\
\text { jusqu'aux genoux avant de solli- } \\
\text { citer de l'aide pour la poser sur la } \\
\text { tête. " }\end{array}$ \\
\hline $\begin{array}{l}\text { Organisations } \\
\text { paysannes }\end{array}$ & $\begin{array}{l}\text { La recherche coûte cher. Mais les producteurs } \\
\text { devraient y apporter une contribution financière. }\end{array}$ & $\begin{array}{l}\text { Les producteurs devraient soutenir } \\
\text { financièrement la recherche et la } \\
\text { vulgarisation agricoles. }\end{array}$ \\
\hline $\begin{array}{l}\text { Projets de } \\
\text { développement }\end{array}$ & $\begin{array}{l}\text { Les bénéficiaires devraient financer la RVA, surtout si } \\
\text { elle concerne des problèmes spécifiques locaux. }\end{array}$ & $\begin{array}{l}\text { Les producteurs sont souvent prêts } \\
\text { et devraient apporter une contri- } \\
\text { bution en nature. }\end{array}$ \\
\hline
\end{tabular}

Source : I'auteur.

financement des producteurs. Il s'agit de la recherche-développement orientée vers la résolution de problèmes concrets, spécifiques et locaux de production agricole. $\mathrm{Au}$ niveau de la plupart des ONG, on estime par exemple que les producteurs devraient assurer la diffusion des produits de recherche arrivés à maturité. Dans le même sens, la plupart des agents de terrain de diverses institutions de RVA estiment qu'une partie du financement devrait provenir des bénéficiaires.

Au demeurant, la plupart des principaux acteurs pensent qu'actuellement les sources de financement de la RVA doivent être diversifiées. Le financement de l'État devrait être renforcé par les institutions internationales, les ONG, les structures locales et les bénéficiaires directs, surtout s'il s'agit de la résolution de problèmes spécifiques locaux. Mais il est important que ces fonds ne soient pas tous accompagnés de conditionnalités et obligations différentes qui compromettent l'efficacité de la RVA.

\section{Quelle participation financière des producteurs?}

L'analyse des interviews des différents acteurs permet de structurer leurs perceptions de la contribution des producteurs autour de trois thématiques principales. Il s'agit (i) de contraintes au financement par les paysans et leurs organisations, (ii) comment augmenter la participation financière 
paysanne ? (iii) quelle contribution peuton espérer des producteurs?

\section{Contraintes au financement par les paysans et leurs organisations}

L'analyse des interviews fait apparaître que pour les acteurs la faible motivation et l'incapacité financière des producteurs sont des contraintes majeures à la PFP.

\section{Faible motivation des producteurs}

Le sentiment est partagé au sein des principaux acteurs de la RVA, y compris les leaders paysans, que les producteurs et les responsables de leurs organisations manquent actuellement de motivation pour contribuer au financement des services (tableau 3).

Les producteurs seraient plus motivés à mobiliser le financement destiné à la construction d'infrastructures sociocommunautaires qui sont des réalisations tangibles. Leur motivation serait suffisante pour apporter des contributions en nature ou des ressources locales en faveur de la RVA. Cette motivation ne serait que théorique lorsque la participation devrait être financière. Elle ne résisterait pas aux initiatives de décaissement de fonds pour soutenir la RVA. Ce qui était jugé très intéressant par les producteurs - quand le financement provient d'ailleurs l'est beaucoup moins lorsque le financement devrait provenir d'eux-mêmes.

\section{Incapacité financière des producteurs}

Les membres du personnel dirigeant des organisations impliquées dans la RVA pensent que la plupart des producteurs disposent de toutes petites exploitations agricoles et produisent principalement pour l'autosubsistance. Leurs revenus sont aléatoires et ne seraient pas suffisants pour financer les services. Par contre, beaucoup d'agents de terrain des ONG, des organisations de producteurs et des projets de développement estiment qu'il est possible de mobiliser au niveau des producteurs un financement en nature ou en espèce s'ils

Tableau 3. Synthèse des perceptions des différents types d'acteurs sur la motivation des producteurs et des responsables de leurs organisations à contribuer au financement de la RVA

\begin{tabular}{l|l|l}
\hline & \multicolumn{1}{|c|}{ Membres du personnel dirigeant } & \multicolumn{1}{c}{ Agents de terrain } \\
\hline $\begin{array}{l}\text { Recherche } \\
\text { publique }\end{array}$ & $\begin{array}{l}\text { La volonté de participer au financement de } \\
\text { la RVA fait défaut au niveau des structures } \\
\text { faîtières des organisations de producteurs. }\end{array}$ & $\begin{array}{l}\text { Les producteurs refusent de financer même } \\
\text { des activités jugées très intéressantes par } \\
\text { eux parce qu'ils pensent que les fonds } \\
\text { devraient provenir d'ailleurs. }\end{array}$ \\
\hline $\begin{array}{l}\text { Vulgarisation } \\
\text { publique }\end{array}$ & $\begin{array}{l}\text { Les producteurs manifestent une volonté } \\
\text { tant qu'on reste au niveau théorique. Mais } \\
\text { on ne sent plus cette volonté dès lors qu'il } \\
\text { est question de décaisser des fonds. }\end{array}$ & $\begin{array}{l}\text { La volonté manque aux responsables des } \\
\text { organisations de producteurs qui semblent } \\
\text { avoir plus d'intérêt pour des actions à } \\
\text { effets beaucoup plus immédiats. }\end{array}$ \\
\hline $\begin{array}{l}\text { ONG } \\
\text { Organisations } \\
\text { paysannes }\end{array}$ & $\begin{array}{l}\text { Les paysans manquent de motivation à finan- } \\
\text { cer les services de vulgarisation agricole. } \\
\text { motivés à financer l'encadrement. }\end{array}$ & $\begin{array}{l}\text { Les producteurs sont souvent prêts à appor- } \\
\text { ter des ressources locales. Mais la participa- } \\
\text { tion financière n'est pas évidente. }\end{array}$ \\
\hline $\begin{array}{l}\text { Projets de } \\
\text { développement }\end{array}$ & $\begin{array}{l}\text { Les producteurs et leurs associations } \\
\text { n'acceptent pas de financer facilement les } \\
\text { services. Ils attendent plutôt que tout pro- } \\
\text { vienne de l'extérieur. }\end{array}$ & $\begin{array}{l}\text { La volonté reste à développer par la sensi- } \\
\text { bilisation des producteurs. }\end{array}$ \\
\hline
\end{tabular}


RECHERCHES

Ismail M. MOUMOUNI

jugent le service intéressant et si la période de récupération des fonds est bien choisie (tableau 4).

Des distinctions sont cependant à effectuer, d'une part entre le financement par les organisations de producteurs et celui par des paysans individuels, et d'autre part entre les gros et les petits producteurs.

- Distinction entre le financement par les organisations de producteurs et par les paysans individuels : les organisations de producteurs surtout faîtières seraient en mesure de participer au financement de la RVA tandis que les producteurs individuels en seraient pour la plupart incapables. De même, en finançant les services agricoles, une organisation de producteurs pourrait être beaucoup plus préoccupée par une augmentation globale de la production, peu importe celui qui aurait produit. Les services pourraient être ainsi orientés vers les gros

Tableau 4. Synthèse des perceptions des différents types d'acteurs sur la capacité des producteurs et de leurs organisations à contribuer au financement de la RVA

\begin{tabular}{|c|c|c|}
\hline & Membres du personnel dirigeant & Agents de terrain \\
\hline Ministère & $\begin{array}{l}\text { La petitesse des exploitations agricoles rend difficile } \\
\text { l'intervention des producteurs dans le financement de } \\
\text { la RVA. }\end{array}$ & - \\
\hline Université & $\begin{array}{l}\text { Certains gros producteurs sont plus ou moins des } \\
\text { exploitants agricoles et peuvent financer des services } \\
\text { dans le cadre de leurs associations. Les petits produc- } \\
\text { teurs de subsistance à revenus limités et aléatoires ne } \\
\text { sont pas prêts à financer les services. }\end{array}$ & - \\
\hline $\begin{array}{l}\text { Recherche } \\
\text { publique }\end{array}$ & $\begin{array}{l}\text { La plupart des utilisateurs des produits de recherche } \\
\text { sont de petits producteurs qui produisent pour } \\
\text { l'autosubsistance. Les revenus issus de la vente partielle } \\
\text { de leurs récoltes ne sont pas assez substantiels pour } \\
\text { financer des services. Mais les organisations faîtières des } \\
\text { producteurs pourraient le faire. }\end{array}$ & $\begin{array}{l}\text { Les producteurs individuels ne } \\
\text { sont pas en mesure de finan- } \\
\text { cer la RVA. Mais les organisa- } \\
\text { tions faîtières sont en mesure } \\
\text { de le faire. }\end{array}$ \\
\hline $\begin{array}{l}\text { Vulgarisation } \\
\text { publique }\end{array}$ & $\begin{array}{l}\text { La plupart des producteurs n'ont pas la capacité finan- } \\
\text { cière pour apporter une contribution au financement } \\
\text { des services de RVA. }\end{array}$ & $\begin{array}{l}\text { - Il faudrait faire une diffé- } \\
\text { rence entre le financement par } \\
\text { les organisations de produc- } \\
\text { teurs et le financement par les } \\
\text { paysans individuels. } \\
\text { - Les producteurs apportent } \\
\text { difficilement les intrants pour } \\
\text { faire des démonstrations. } \\
\end{array}$ \\
\hline ONG & $\begin{array}{l}\text { À l'étape où nous en sommes, les revenus des produc- } \\
\text { teurs sont suffisamment bas pour qu'on leur demande } \\
\text { de financer la recherche et la vulgarisation. L'agricul- } \\
\text { ture telle que pratiquée n'est pas si rentable. }\end{array}$ & $\begin{array}{l}\text { Les paysans n'ont pas toujours } \\
\text { de l'argent. Pendant la } \\
\text { période de paiement des reve- } \\
\text { nus du coton, il est possible } \\
\text { de mobiliser ses ressources } \\
\text { financières pour la RVA. }\end{array}$ \\
\hline $\begin{array}{l}\text { Organisations } \\
\text { paysannes }\end{array}$ & $\begin{array}{l}\text { La RVA coûte cher. Les producteurs ne pourraient sup- } \\
\text { porter que très partiellement son coût. }\end{array}$ & $\begin{array}{l}\text { Les producteurs ont la capacité } \\
\text { d'apporter une contribution } \\
\text { financière. }\end{array}$ \\
\hline $\begin{array}{l}\text { Projets de } \\
\text { développement }\end{array}$ & $\begin{array}{l}\text { Les revenus des producteurs sont si bas qu'ils ne } \\
\text { peuvent pas penser à acheter de la technologie. }\end{array}$ & $\begin{array}{l}\text { Les producteurs pourraient } \\
\text { apporter une contribution en } \\
\text { nature. }\end{array}$ \\
\hline
\end{tabular}


producteurs. Par contre, le producteur individuel n'accepterait de financer que si les services sont vraiment adéquats par rapport à ses besoins à lui. Les intérêts du producteur individuel ne coïncident donc pas toujours avec ceux de son organisation.

- Distinction entre gros et petits producteurs : certains gros producteurs peuvent financer les services de RVA, notamment pour s'adapter aux conditions d'exportation et être plus compétitifs sur le marché régional et international.

\section{Comment augmenter la participation financière paysanne?}

Selon la plupart des acteurs, l'augmentation de la PFP devrait passer par la prise en compte effective de leurs besoins par la RVA, un changement organisationnel et comportemental au niveau des producteurs et une organisation et bonne gestion des filières porteuses.

\section{Prise en compte des besoins réels des producteurs par la RVA}

La question du niveau de qualification des agents de vulgarisation et de la qualité des services proposés aux producteurs a été posée en ces termes par un producteur d'ananas : «Si nous pouvons trouver quelqu'un qui soit en mesure de comprendre réellement nos problèmes, de nous suivre dans toutes nos activités, de nous aider à résoudre nos problèmes de production, de technologies, de crédits, d'intrants et surtout de commercialisation, nous pouvons nous associer pour l'engager et le rémunérer. » Comme l'ont aussi mentionné la plupart des leaders paysans, pour motiver les producteurs à financer la RVA, il faudrait leur fournir des services dont ils ont réellement besoin. Les membres du personnel dirigeant des projets de développement pensent qu'il faudrait faire en sorte que les paysans voient concrètement leurs intérêts dans les activités de
RVA. Selon beaucoup d'agents de terrain de différentes institutions, il faudrait rendre plus effective l'approche participative dans la RVA. Un responsable des services de vulgarisation publique nous confiait : «J'ai appris seulement quelques années avant ma retraite que beaucoup de producteurs utilisent des technologies simplement pour faire plaisir aux vulgarisateurs. » Donc, en dépit de tous les efforts effectués dans ce sens, beaucoup de services offerts aux producteurs semblent ne pas répondre vraiment à leurs besoins.

Nécessité d'un changement organisationnel et comportemental au niveau des producteurs

Des facteurs organisationnels et comportementaux semblent avoir des conséquences négatives sur la capacité des producteurs à mobiliser des ressources suffisantes pour investir dans la RVA. Des changements seraient requis selon les acteurs, pour augmenter la capacité et la volonté de financement des producteurs.

- Changement socio-organisationnel : il y a aujourd'hui une crise de confiance des producteurs envers certaines de leurs organisations parce que le transfert de compétence survenu entre les organisations publiques et de producteurs a été suivi d'une mauvaise gestion des ressources des producteurs. Les organisations n'étaient pas suffisamment bien préparées pour assumer les responsabilités qui leur avaient été confiées, notamment en ce qui concerne la distribution des intrants et la commercialisation primaire du coton. Cette mauvaise gestion a eu pour conséquence le non-paiement aux producteurs d'une partie des sommes dues au titre de la vente du coton pendant plusieurs années. Les mécanismes de compte rendu et les systèmes d'autocontrôle devraient être renforcés au sein des organisations de producteurs. 
RECHERCHES

Ismail M. MOUMOUNI

Tableau 5. Synthèse des perceptions des différents types d'acteurs sur l'importance de l'organisation des filières porteuses dans la participation des producteurs au financement de la RVA

\begin{tabular}{|c|c|c|}
\hline & Membres du personnel dirigeant & Agents de terrain \\
\hline Université & $\begin{array}{l}\text { Les producteurs ne se rendent compte de l'impor- } \\
\text { tance de l'innovation, de l'information et de la } \\
\text { formation que lorsqu'il y a un vaste marché auquel } \\
\text { ils n'ont pas facilement accès. Ils peuvent encore être } \\
\text { très réticents lorsque le marché demeure incertain. }\end{array}$ & - \\
\hline $\begin{array}{l}\text { Vulgarisation } \\
\text { publique }\end{array}$ & $\begin{array}{l}\text { L'organisation de la filière est indispensable pour } \\
\text { que les producteurs soient en mesure de financer les } \\
\text { services. }\end{array}$ & $\begin{array}{l}\text { Même l'organisation des filières } \\
\text { n'est pas suffisante pour deman- } \\
\text { der aux pauvres producteurs de } \\
\text { financer les services. }\end{array}$ \\
\hline ONG & $\begin{array}{l}\text { II faudrait cibler et bien organiser des options réel- } \\
\text { lement porteuses pour que les producteurs puissent } \\
\text { financer les services. }\end{array}$ & - \\
\hline $\begin{array}{l}\text { Organisations } \\
\text { paysannes }\end{array}$ & $\begin{array}{l}\text { Pour inciter le producteur à financer, il faudrait } \\
\text { œuvrer pour l'amélioration de la production et de la } \\
\text { commercialisation. Mais cette dernière est souvent } \\
\text { occultée. }\end{array}$ & $\begin{array}{l}\text { Si le marché est garanti, le pro- } \\
\text { ducteur peut accepter de financer } \\
\text { la RVA. }\end{array}$ \\
\hline $\begin{array}{l}\text { Projets de } \\
\text { développement }\end{array}$ & $\begin{array}{l}\text { Il faudrait désormais assister le producteur dans } \\
\text { toutes ses activités, depuis la production jusqu'à la } \\
\text { commercialisation. }\end{array}$ & $\begin{array}{l}\text { Sans une politique de commercia- } \\
\text { lisation appropriée, les produc- } \\
\text { teurs ne pourront pas adopter } \\
\text { durablement les technologies ou } \\
\text { financer les services. }\end{array}$ \\
\hline
\end{tabular}

Source : l'auteur.

- Changement de comportement : le versement aux producteurs d'indemnités ou de rémunérations pour des travaux de recherche ou de formation les aurait progressivement détournés de l'intérêt du contenu des activités de RVA vers les sommes versées. Des actions de sensibilisation devraient être conduites en vue de changer un tel comportement.

\section{Organisation et bonne gestion de filières agricoles}

L'expérience de la filière coton révèle, selon la plupart des acteurs, que seule une bonne organisation de filières peut permettre l'amélioration des revenus et des conditions de vie des producteurs; toute chose nécessaire à leur participation au financement de la RVA (tableau 5).

Une minorité des acteurs pense que même l'organisation des filières n'est pas suffisante pour demander aux producteurs pauvres de financer les services agricoles. Mais de façon générale, l'organisation des filières apparaît comme un point central dans la création de conditions favorables à la participation des producteurs au financement de la RVA. Dans cette organisation, l'existence et l'assurance d'un marché rémunérateur, et les facilités de commercialisation, apparaissent aux acteurs comme la clé du succès. Au Sud-Bénin, des exportateurs d'ananas endossent les petits producteurs en leur permettant l'accès au marché. Cela motive plus d'un producteur à produire de l'ananas pour l'exportation. Cette expérience montre bien que le suivi du producteur jusqu'à la commercialisation semble donc nécessaire pour l'encourager à participer au financement des services agricoles. Une bonne organisation de filière devrait prendre aussi en compte l'accès aux intrants et au crédit, et une gestion transparente des ressources. 
Pour que les producteurs et leurs associations soient plus enclins à contribuer au financement des services, il faudrait que des filières soient mieux organisées et que cette meilleure organisation se traduise par une amélioration de leurs revenus. $\mathrm{Si}$ le producteur constate que les services de RVA apportent une véritable valeur ajoutée, il acceptera plus facilement de contribuer à leur financement. Mais cette contribution devrait être prélevée sur le surplus de revenus engendré par les interventions de la RVA afin d'éviter une paupérisation des producteurs. «Le producteur n'achète pas le poisson dans l'eau. » Cette perception n'est cependant pas celle de tous les acteurs. Certains agents des institutions publiques estiment qu'il y a aujourd'hui des exemples qui montrent l'utilité de la RVA au Bénin. Elle a contribué à l'amélioration de la production de plusieurs cultures telles que le coton et le manioc.

\section{Quelle contribution des producteurs ?}

Tous les acteurs rencontrés pensent que le paiement individuel direct sous forme d' « argent chaud » qui devrait augmenter le niveau de responsabilité, de vigilance et de contrôle des producteurs par rapport aux services de RVA (Katz, 2002) par les producteurs n'est pas envisageable. Les producteurs pourraient accepter le principe de prélèvement, mais pas celui du paiement direct. À ce sujet, un producteur de coton déclarait : "Quand l'argent rentre dans la poche du producteur, il ne sort plus facilement pour retourner aux services d'encadrement. » De plus, selon beaucoup d'agents de terrain, avant que les producteurs ne soient en mesure d'exercer un droit d'influence sur les services de RVA, il faudrait les y former et les y habituer. Cependant, il est admis par certains que la participation paysanne individualisée améliorerait la qualité des prestations. Il y aurait beaucoup moins d'agents car beaucoup seraient renvoyés du fait de l'inanité de leurs services. De même, beaucoup d'interventions se révéleraient inutiles. Ces interventions seraient moins systématiques et plus liées aux contextes spécifiques. Elles seraient donc mieux centrées sur les besoins ponctuels individuels.

\section{Discussion}

Les résultats de cette étude permettent d'examiner les relations entre, d'une part, la participation financière des producteurs et l'appropriation des services, et d'autre part, le caractère privé d'un service et la participation financière des producteurs.

\section{Participation financière des producteurs et appropriation des services}

Les discours habituels sur le financement de la RVA arguent que l'introduction de la PFP vise à renforcer la position des producteurs et à développer un sens des responsabilités de façon à amener les organismes de RVA à améliorer la qualité de leurs prestations (Rivera et al., 2001 ; Katz, 2002 ; Groupe de Neuchâtel, 1999). La PFP est donc supposée être un moyen pouvant amener les prestataires à orienter leurs services vers les besoins des producteurs et à rendre compte de leurs activités (Collion et Rondot, 1998 ; Ban, 2000). Les analyses de perceptions révèlent que les acteurs locaux du développement à divers niveaux de hiérarchie professionnelle perçoivent différemment la PFP. Elle est perçue beaucoup moins par ces derniers comme une manière d'augmenter le sens des responsabilités des producteurs et le niveau de contrôle exercé par les producteurs sur les institutions de service. Plutôt, la PFP vise beaucoup plus la mobilisation de ressources complémentaires pour assurer le fonctionnement de la RVA. Cela explique le fait que des producteurs 
financent des institutions de service par le biais de leur organisation faîtière - dont les leaders sont bien souvent éloignés géographiquement. Les résultats de cette étude indiquent aussi que, même en cas de participation financière directe, il n'est pas évident ou automatique que les producteurs s'engagent dans une opération de contrôle. Le sens et la capacité de contrôler une institution de service, si nécessaire, doivent être développés et renforcés progressivement.

\section{Caractère privé d'un service et participation financière des producteurs}

Umali et Schwartz (1994) suggèrent une classification des biens et services suivant leur caractère privé ou public (possibilités d'exclusion et de rivalité). Le niveau de la PFP et de la contribution de tous les acteurs dépendrait donc du ratio d'intérêt privé/public. Les perceptions des acteurs locaux et nationaux rejoignent Umali et Schwartz sur la nécessité de combiner plusieurs sources de financement de la RVA. La divergence se situe au niveau de l'importance du caractère privé d'un service dans la détermination de la source de financement appropriée. Notre étude montre que la PFP dépend de plusieurs facteurs autres que technologiques qui sont pour une large part spécifiques aux conditions locales. Il s'agit notamment (i) des facteurs socio-économiques, (ii) des facteurs organisationnels, et (iii) des expériences des producteurs liées à l'organisation et au financement de la RVA. Ainsi, le caractère privé d'un service de RVA semble ne pas être suffisant pour constituer une motivation. Les acteurs locaux et nationaux considèrent plutôt l'utilité pratique des services pour se déterminer à les financer. Lorsqu'un service est perçu par les producteurs comme utile, la question ne semble pas se poser en termes d'excludability, mais plutôt en termes d'includability, c'est-à-dire la possibilité pour les producteurs de s'associer pour participer au financement.

$$
\text { * }
$$

Il ressort des résultats que la mobilisation du financement de la RVA est une préoccupation partagée par la plupart des principaux acteurs. Selon les acteurs locaux et nationaux, le financement de la RVA devrait principalement provenir de l'État dans le cadre de la mise en œuvre de sa politique de développement agricole et pour assurer une certaine souveraineté. Les bénéficiaires devraient aussi apporter une contribution sous certaines conditions qui sont (i) l'amélioration et la réorientation des compétences en ressources humaines pour la satisfaction des besoins réels des producteurs, (ii) l'organisation et la bonne gestion de filières agricoles, et (iii) une contribution paysanne prélevée sur le surplus de revenus engendrés par les services afin d'éviter une paupérisation des producteurs. Il s'avère aussi nécessaire de sensibiliser les bénéficiaires pour un changement progressif de la logique selon laquelle le financement de la RVA doit nécessairement provenir de l'extérieur ou de l'État. De ces résultats ressortent la nécessité de la mise en place d'un cadre de concertation entre les acteurs impliqués dans l'organisation et le financement de la RVA, comme souligné par Farrington (1994), Carney (1998), Rivera et Zijp (2000), Kidd et al. (2000) et Barandun (2001). Enfin, il s'en dégage que la PFP ne conduit pas forcément à une appropriation des services par les producteurs. 


\section{RÉFÉRENCES BIBLIOGRAPHIQUES}

Ban van den A.W. (2000). Different ways of financing agricultural extension. Agricultural Research and Extension Network, ${ }^{\circ} 106$, p. 8-12.

Ban van den A.W., Brouwers H.S., Boon C.M. (1994). La vulgarisation agricole en Afrique. Paris, Karthala, Centre technique de coopération agricole et rurale.

Barandun A. (2001). More accountability of extension providers through financial reforms and services market: A summury of our internet debate in the year 2000. BeraterInnen News, 1/2001.

Carney D. (1998). Changing public and private roles in agricultural service provision. London, Overseas Development Institute, Natural Resources Group.

Collion M.H., Rondot P. (1998) Partnerships between agricultural services institutions and producers' organizations: myth or reality? Agricultural Research and Extension Network Paper, n80, London, ODI.

Farrington J. (1994). Public sector agricultural extension: Is there life after structural adjustment. London, ODI.

Groupe de Neuchâtel (1999). Note de cadrage sur la vulgarisation agricole. Paris, Groupe de Neuchâtel.

Groupe de Neuchâtel (2002). Note de cadrage sur le financement du conseil agricole et rural. Lindau, Groupe de Neuchâtel.

INRAB/MAEP (2004). Plan d'action de la recherche agricole nationale. Cotonou, deuxième version.

Katz E. (2002). Innovative approaches to financing extension for agriculture and natural resource management. Eschikon, LBL.

Kidd A.D, Lamers J., Ficarelli P., Hoffmann V. (2000). Privatising agricultural extension: cavear emptor. Journal of Rural Studies, $\mathrm{n}^{\circ} 16$, p. 95-102.

Klerkx L., Leeuwis C. (2009). Shaping Collective Functions in Privatized Agricultural Knowledge and Information Systems: The Positioning and Embedding of a Network Broker in the Dutch Dairy Sector. The Journal of Agricultural Education and Extension, vol. $15, \mathrm{n}^{\circ} 1$, p. 81-105.
MAEP (2001). Plan stratégique opérationnel. Cotonou.

MAEP (2004). Plan d'action national formation et vulgarisation. Cotonou, Version provisoire après l'atelier de validation du 10 août 2004.

MDR (1991). Lettre de déclaration de politique de développement rural. Washington.

MDR (1999). Déclaration de la politique de développement rural. Cotonou.

Long N. (1992). From Paradigm Lost to Paradigm Regained: The case of An Actororiented Sociology of Development. In Long N., Long A. (eds.), Battlefields of Knowledge, The Interlocking of Theory and Practice in Social Research and Development. London, Routledge, p. 16-46.

Long N. (2003). An Actor-oriented Approach to Development Intervention. In Cruz D.A. (ed.), Rural life improvement in Asia, Japan, Report of the APO Seminar on Rural Life Improvement for Community Development, 22-26 April 2002.

Moumouni M.I. (2007). Impacts of Privatisation of Advisory Services on Agricultural Knowledge and Information Systems: Some Evidences from "LEC" Knowledge Management in Banikoara. Benin, Quarterly Bulletin of the International Association of Agricultural Information Specialists, vol. 51, n³/4, p. 208-214.

Nouatin S.G. (2003). Dynamiques de la participation au Bénin : rôles et stratégies de différents groupes d'intérêt des processus de recherche agricole. Kommunikation und Beratung- Sozialwissenschaftliche Schriften zur Landnutzung und ländlichen Entwicklung 58. Weikersheim, Margraf Verlag.

Rivera W.M., Qamar K.M., Van Crowder L. (2001). Agricultural and Rural Extension Worldwide: Options for Institutional Reform in the Developing Countries. FAO.

Rivera M.W., Cary J.W. (1997). Privatising agricultural Extension. In Swanson E.; Bentz R.P., Sofrando A.J. (eds.), Improving agricultural extension: A Reference manual, Roma, FAO. 
RECHERCHES

Ismail M. MOUMOUNI

Rivera M.W., Zijp W. (2000). Contracting for extension services: An International Perspective. In AIAEE (ed.), 2000 Conference Proceeding, $16^{\text {th }}$ Annual Conference, Arlington, March $29^{\text {th }}$-April $1^{\text {st }} 2000$, p. 1-6.

Schmidt P., Etienne C., Hurlimann M. (1998). Vulgarisation participative : aperçu de trois projets de développement agricole en Afrique. DDC, SKAT and LBL, Lindau.

Umali D.L., Schwartz L. (1994). Public and Private agricultural extension: Beyond traditional frontiers. Washington D.C., World Bank Discussion, Paper $n^{\circ} 236$. 